\title{
DIFFICULT AIRWAY IN A PATIENT WITH LYMPHOMA
}

\section{Authors: E. González-Benito', T. Del Castillo',P. Cruz ${ }^{1}, I$. Solchaga ${ }^{1}, S$. Díaz ${ }^{1}, M$. López-Gil ${ }^{1}$ Department of Anesthesia. Gregorio Marañón University Hospital ${ }^{1}$}

\section{BACKGROUND}

Unanticipated difficult airway management is a challenge for every anesthesiologist. We report the case of a patient with Waldeyer's ring hypertrophy secondary to a lymphoma that resulted in difficult airway management. This fact remained unnoticed in preoperative assessment.

\section{CASE REPORT}

A 86-year-old male patient, ASA III, diagnosed with lymphoma, was scheduled for a submaxillectomy to choose his best chemotherapy treatment. He had a medical history of hypertension, ischemic heart disease an lacunar infarct, without any surgical history. He referred nasal voice and recent laterocervical adenopathies without respiratory symptoms. Following anesthesic induction, mask ventilation was impossible (Han IV). First direct laryngoscopy was done, observing Cormack-Lehane Grade III, severe anatomic distortion and bleeding. After that, a more skilled anesthesiologist performed two videolaryngoscopy attempts without any vision improvement. A suppraglottic airway device was inserted and ventilation obtained. Succesful intubation was achieved by performing a fiberoptic bronchoscopy through the supraglottic device although glotis could not be correctly observed. Delayed extubation was considered to be the safest option and patient was transferred safely to the Reanimation Unit. There were no incidences in extubation. TC imaging evaluated after the procedure revealed a severe narrowing of parapharyngeal space due to Waldeyer's ring hypertrophy.

\section{DISCUSSION}

Waldeyer's ring is a lymphoid structure that surrounds pharynx. It is a frecuent extranodal non-Hodgkin lymphoma's location. However, its hypopharyngeal involvement is an uncommon difficult airway's ethiology so it may go unnoticed in preoperative assessment, as happened to us. Therefore, imaging test, especially TC scan, may reveal it and should always be performed and carefully evaluated in order to elaborate a proper therapeutic approach. After induction, loss of pharyngeal muscles tone can impair airway's patency and cause upper airway obstruction. Moreover, pharyngeal pathology hinders fibrobronchoscopic and videolaryngoscopic intubation. Hence, elaborating an airway strategy management based on limiting airway manipulation and

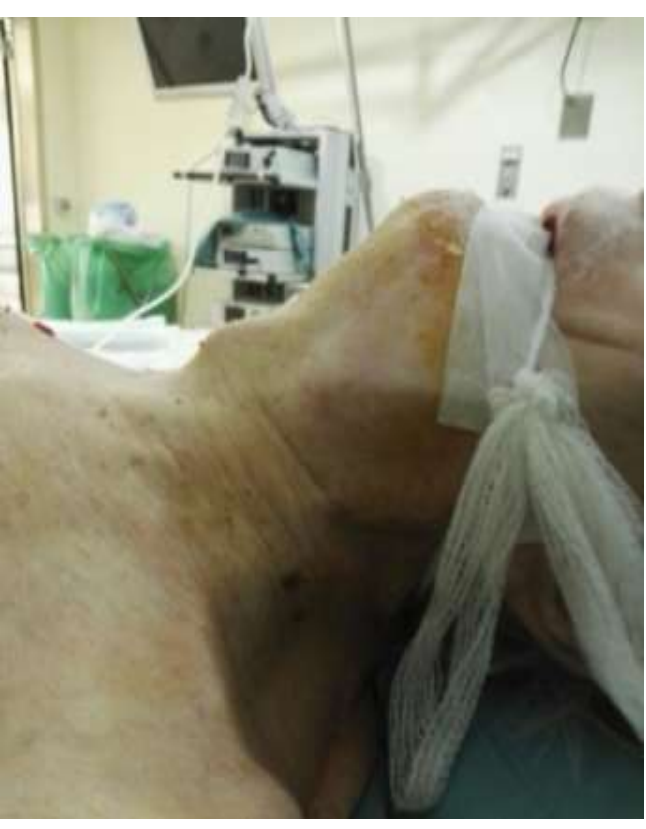
priorizing oxigenation is compulsory.
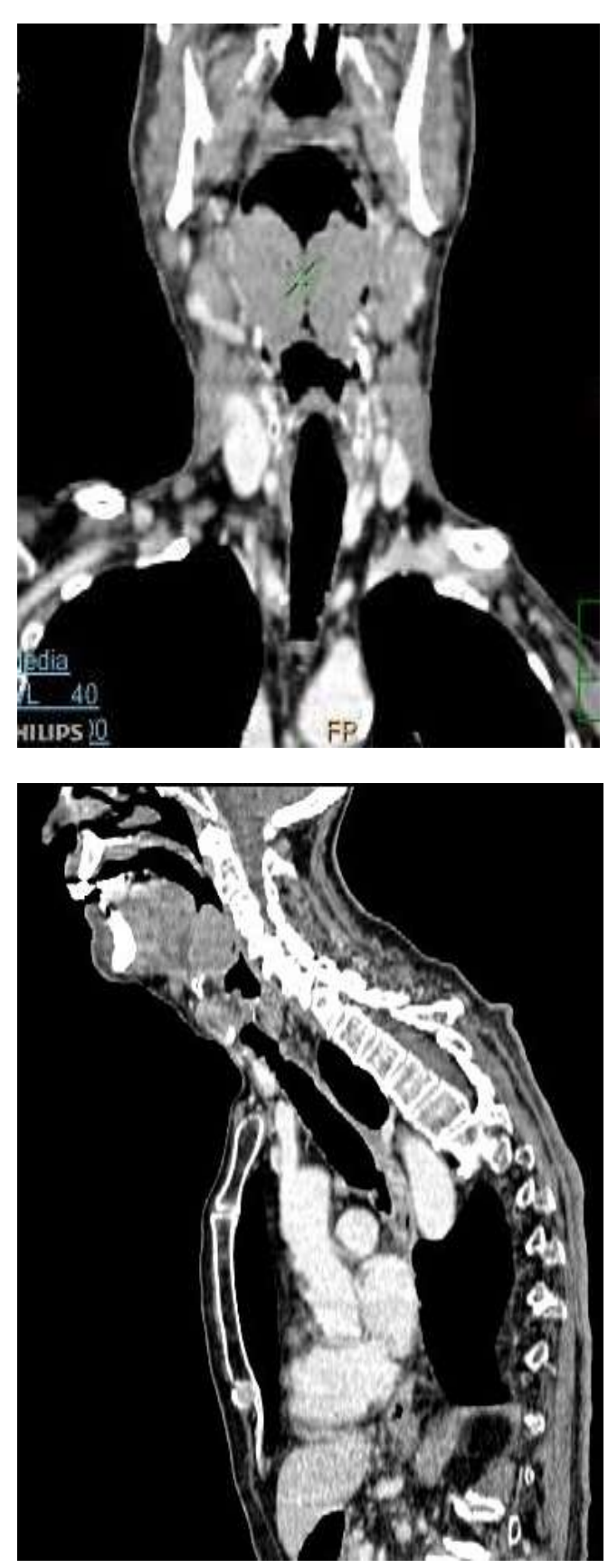

\section{LEARNING POINTS}

1. Advanced lymphoproliferative syndromes cause disseminated adenopathies whose pharyngeal involvement can lead to a difficult airway. Absence of risk predictors does not exclude it.

2. Multi-slice detector computed tomography is considered as the gold standard image technique.

3. We should always elaborate an adequate airway strategy based on an unanticipated difficult intubation algorithm.

4. Limiting airway manipulation and priorizing oxigenation are mandatory . 